\title{
World Health Organization infant and young child feeding indicators and their associations with child anthropometry: a synthesis of recent findings
}

\author{
Andrew D. Jones*, Scott B. Ickes ${ }^{\dagger}$, Laura E. Smith*, Mduduzi N.N. Mbuya ${ }^{\ddagger}$, \\ Bernard Chasekwa ${ }^{\ddagger}$, Rebecca A. Heidkamp $^{\S}$, Purnima Menon $\rrbracket$, Amanda A. Zongrone* \\ and Rebecca J. Stoltzfus* \\ *Division of Nutritional Sciences, Cornell University, Ithaca, New York, USA, 'Department of Kinesiology and Health Sciences, College of William and Mary, \\ Williamsburg, Virginia, USA, `ZVITAMBO Project, Harare, Zimbabwe, ${ }^{\circledR}$ The Johns Hopkins University, Baltimore, Maryland, USA, and "Poverty, Health and \\ Nutrition Division, International Food Policy Research Institute, New Delhi, India
}

\begin{abstract}
As the World Health Organization (WHO) infant and young child feeding (IYCF) indicators are increasingly adopted, a comparison of country-specific analyses of the indicators' associations with child growth is needed to examine the consistency of these relationships across contexts and to assess the strengths and potential limitations of the indicators. This study aims to determine cross-country patterns of associations of each of these indicators with child stunting, wasting, height-for-age $z$-score (HAZ) and weight-for-height $z$-score (WHZ). Eight studies using recent Demographic and Health Surveys data from a total of nine countries in sub-Saharan Africa (nine), Asia (three) and the Caribbean (one) were identified. The WHO indicators showed mixed associations with child anthropometric indicators across countries. Breastfeeding indicators demonstrated negative associations with HAZ, while indicators of diet diversity and overall diet quality were positively associated with HAZ in Bangladesh, Ethiopia, India and Zambia $(P<0.05)$. These same complementary feeding indicators did not show consistent relationships with child stunting. Exclusive breastfeeding under 6 months of age was associated with greater WHZ in Bangladesh and Zambia $(P<0.05)$, although CF indicators did not show strong associations with WHZ or wasting. The lack of sensitivity and specificity of many of the IYCF indicators may contribute to the inconsistent associations observed. The WHO indicators are clearly valuable tools for broadly assessing the quality of child diets and for monitoring population trends in IYCF practices over time. However, additional measures of dietary quality and quantity may be necessary to understand how specific IYCF behaviours relate to child growth faltering.
\end{abstract}

Keywords: infant and young child feeding, WHO feeding indicators, diet diversity, child growth, stunting, wasting.

Correspondence: Dr Andrew D. Jones, 313 Savage Hall, Ithaca, NY 14853, USA. E-mail: adj23@cornell.edu

\section{Introduction}

Children who are poorly nourished in the first 2 years of life are at increased risk of mortality and impaired cognitive development, as well as diminished work capacity and chronic disease later in life (Dewey \& Begum 2011). Proper care for children is an essential element needed for the healthy growth and develop- ment of a child (Engle et al. 1996). A major component of care is the set of practices caregivers employ to provide breast milk and complementary foods to children in their first years of life (PAHO/WHO 2003). Relatively simple indicators for assessing breastfeeding $(\mathrm{BF})$ practices have been in widespread use since the early 1990s (World Health Organization 1991). The ability to measure and monitor these 
Table I. World Health Organization infant and young child feeding core indicators

\begin{tabular}{|c|c|}
\hline Indicator & Description \\
\hline \multicolumn{2}{|l|}{ Breastfeeding indicators } \\
\hline 1. Early initiation of breastfeeding & Proportion of children born in the last 24 months who were put to the breast within $1 \mathrm{~h}$ of birth \\
\hline $\begin{array}{l}\text { 2. Exclusive breastfeeding under } \\
6 \text { months }\end{array}$ & $\begin{array}{l}\text { Proportion of infants } 0-5 \text { months of age who were fed exclusively breast milk during the previous } \\
\text { day }\end{array}$ \\
\hline $\begin{array}{l}\text { 3. Continued breastfeeding at } \\
1 \text { year }\end{array}$ & $\begin{array}{l}\text { Proportion of children } 12-15 \text { months of age who were fed any breast milk during the previous } \\
\text { day }\end{array}$ \\
\hline \multicolumn{2}{|l|}{ Complementary feeding indicators } \\
\hline $\begin{array}{l}\text { 4. Introduction of solid, semi-solid } \\
\text { or soft foods }\end{array}$ & $\begin{array}{l}\text { Proportion of infants } 6-8 \text { months of age who received solid, semi-solid or soft foods during the } \\
\text { previous day }\end{array}$ \\
\hline 5. Minimum dietary diversity & $\begin{array}{l}\text { Proportion of children } 6-23 \text { months of age who received foods from } 4 \text { or more food groups } \\
\text { during the previous day }\end{array}$ \\
\hline 6. Minimum meal frequency & $\begin{array}{l}\text { Proportion of breastfed and non-breastfed children } 6-23 \text { months of age who received solid, } \\
\text { semi-solid or soft foods (but also including milk feeds for non-breastfed children) the minimum } \\
\text { number of times or more during the previous day }\end{array}$ \\
\hline 7. Minimum acceptable diet & $\begin{array}{l}\text { Proportion of children 6-23 months of age who had at least the minimum dietary diversity and } \\
\text { minimum meal frequency (apart from breast milk) during the previous day }\end{array}$ \\
\hline $\begin{array}{l}\text { 8. Consumption of iron-rich or } \\
\text { iron-fortified foods }\end{array}$ & $\begin{array}{l}\text { Proportion of children } 6-23 \text { months of age who received an iron-rich food or iron-fortified food } \\
\text { that is specially designed for infants and young children, or that is fortified in the home during } \\
\text { the previous day }\end{array}$ \\
\hline
\end{tabular}

practices has helped raise awareness of the importance of BF and has facilitated progress in achieving improvements in BF practices worldwide (UNICEF 2006). Yet, despite an ever-growing evidence base supporting the importance of complementary feeding (CF) beginning at 6 months of age, defining simple CF indicators has proven challenging because of the multiple dimensions of $\mathrm{CF}$, the variation in these practices across contexts and the changes in recommended practices that occur from 6 to 23 months of age (Arimond et al. 2008; World Health Organization 2008).

In 2008, the World Health Organization (WHO) published a set of population-level IYCF indicators developed in response to the need for simple, practi- cal indicators of appropriate feeding practices in children aged 6-23 months that could be developed from large-scale survey data to describe trends over time and allow for national and sub-national comparisons not only of BF practices but also CF practices (World Health Organization 2002, 2008). A core set of eight indicators (i.e. three $\mathrm{BF}$ and five $\mathrm{CF}$ ) include measures of dietary diversity, feeding frequency, consumption of iron-rich or iron-fortified foods as well as indicators of appropriate BF practices (Table 1). All of these indicators can be constructed from the existing data collected in surveys such as the Demographic and Health Surveys (DHS) (Arimond et al. 2008; ICF Macro 2011). An examination of these practices in 46 countries for which DHS data were available between

\section{Key messages}

- The World Health Organization (WHO) infant and young child feeding (IYCF) indicators are simple measures of diet quality with many applications.

- WHO breastfeeding indicators are negatively associated with child height-for-age z-score (HAZ) in many countries, suggesting that these relationships may be prone to reverse causality.

- Indicators of diet quality were positively associated with HAZ in some countries, although child stunting was not consistently associated with these indicators.

- The WHO IYCF indicators lack specificity, and therefore, additional measures of diet quality may be useful for understanding how specific behaviours are associated with child growth. 
2002 and 2008 revealed inadequate CF practices among large proportions of respondents, particularly in sub-Saharan Africa (SSA) and South Asia (World Health Organization 2010a). In 11 of the 27 SSA countries analysed, fewer than $30 \%$ of children aged 6-23 months achieved a minimally diverse diet, while in no SSA country did greater than $30 \%$ of children achieve a minimally acceptable diet according to the WHO indicators.

Previous dietary diversity scores and child feeding indices have been used to assess the quality of IYCF practices in different country contexts and to examine the association of these practices with child anthropometry (Hatløy et al. 2000; Arimond \& Ruel 2002; Ruel \& Menon 2002; Savy et al. 2005). These studies have generally found that proper child feeding practices are associated with a lower probability of child stunting (i.e. low height-for-age), an indicator of chronic undernutrition. One recent study pooled data from 14 low-income countries to assess the overall average effect of the WHO IYCF indicators on child anthropometry across all countries rather than measuring the impacts for individual countries (Marriott et al. 2012). The authors found that all of the WHO CF indicators, with the exception of the indicator defining minimum meal frequency (MMF), were associated with a significantly lower probability of a child being underweight (i.e. low weight-for-age) and stunted.

Understanding the extent to which indicators of dietary quality predict anthropometric outcomes is important for interpreting the meaning of the measurements arising from these indicators. Especially because the WHO IYCF indicators are meant to be used not only for describing trends in IYCF practices over time but also for identifying populations at risk and evaluating the impacts of interventions, the indicators may not achieve their intended purpose if they do not predict the ultimate outcomes of interest for assessing nutritional risk or impact, namely child growth and development. However, the strength and relevance of an indicator depend on the purpose for which it is intended (Habicht \& Pelletier 1990). Individual-level associations with child anthropometry may be unimportant for assessing the relevance of an indicator if the inferences that need to be drawn from the data are not at the individual level, but rather are at the national or sub-national level (e.g. comparing trends across countries or regions), or are not directly concerned with child anthropometric outcomes (e.g. drawing attention to disparities in child feeding practices for advocacy purposes).

It is only recently that analyses have emerged examining associations between the gamut of the WHO recommended IYCF indicators and child anthropometry. As these indicators are increasingly used in programmatic and research settings to inform assessment, targeting, and monitoring and evaluation efforts, analyses of the associations between these indicators and child anthropometric outcomes are needed to develop an understanding of the strengths and potential limitations of the indicators when used for different purposes. This understanding then may lead to a more informed process for selecting indicators and metrics of child diets and feeding practices in different contexts. Furthermore, country-specific analyses of the relationships between these IYCF indicators and child anthropometry are also needed. Pooling data across countries increases statistical power and improves precision for estimating average effects; however, it may also mask important variability across countries (e.g. feeding trends in a subgroup of countries may disproportionately drive the average effects observed).

We review here the current literature examining the eight core WHO IYCF indicators and their relationships with child anthropometry using countrylevel data. Our objectives are (1) to examine the patterns of associations across countries of each of the indicators with child stunting and wasting as well as child height-for-age $z$-score (HAZ) and weight-forheight $z$-score (WHZ); (2) to assess the consistency of the relationships observed across countries; and based on these analyses, (3) to develop an understanding of the strengths and potential limitations of the indicators when used for different purposes.

\section{Materials and methods}

\section{Study selection}

We conducted a literature search to identify peerreviewed journal articles that examined associations 
between at least one of the WHO IYCF indicators and at least one of the following child anthropometric outcomes: stunting, wasting, HAZ or WHZ. We searched Google Scholar, PubMed Central and Web of Science for relevant articles from 2008 to 2013, reviewing the titles and abstracts of all identified studies. All citations from relevant articles were examined for additional references and we identified several yet-to-be-published articles through personal contacts with expert colleagues. Articles that examined relationships between IYCF practices and child anthropometry, but did not construct measures of child feeding using the definitions recommended by the WHO (World Health Organization 2008), were not included.

\section{Measurement of variables}

All of the studies reviewed used DHS data as their data source. The DHS model survey instruments changed in many countries in 2008 from phase 5 questionnaires (used from 2003 to 2008) to phase 6 questionnaires (used from 2008 to 2013). Only the Kenya and Zimbabwe data presented here were collected using phase 6 questionnaires. Data from all other countries were collected using phase 5 questionnaires.

We review data only on the eight core WHO indicators (Table 1). Early initiation of breastfeeding (EIB) is based on historic recall by mothers of children born in the last 24 months who were put to the breast within $1 \mathrm{~h}$ of birth. All other indicators are based on recall of practices from the previous day. For example, exclusive breastfeeding (EBF) among children 0-5 months of age and continued breastfeeding at 1 year (CBF) among children 12-15 months of age are calculated from recall of exclusive and any BF, respectively, the previous day only. Introduction of solid, semi-solid or soft foods (ISF) is the proportion of infants 6-8 months of age who received solid, semisolid or soft foods the previous day. The minimum dietary diversity (MDD) indicator is calculated based on consumption of at least four of the following seven food groups: (1) grains, roots and tubers; (2) legumes and nuts; (3) dairy products; (4) flesh foods; (5) eggs; (6) vitamin A-rich fruits and vegetables; and (7) other fruits and vegetables. Breastfed infants aged 6-8 and
9-23 months must have received solid, semi-solid or soft foods at least two times and three times, respectively, in the previous day to achieve the MMF. Nonbreastfed children aged 6-23 months must receive solid, semi-solid or soft foods or milk feeds at least four times in the previous day to achieve the MMF. The minimum acceptable diet (MAD) indicator is a composite of the MDD and MMF indicators. Breastfed children aged 6-23 months must have had the MDD and MMF the previous day, while nonbreastfed children aged 6-23 months must have received at least two milk feedings and had at least the MDD (not including milk feeds) and the MMF during the previous day. The consumption of iron-rich or iron-fortified foods (IRF) indicator is defined as children 6-23 months of age who received, during the previous day, an iron-rich food or a food that was specially designed for infants and young children and was fortified with iron.

All identified studies calculated child anthropometric measures based on the WHO Child Growth Standards (World Health Organization 2010b) and among those studies reporting stunting and wasting data, $z$-score cut-off points of $\leq 2$ SD were used to classify low height-for-age (i.e. stunting) and low weight-forheight (i.e. wasting).

\section{Heterogeneity in model specification}

We report on regression coefficients, $P$-values and $95 \%$ confidence intervals around point estimates from multiple linear regression models that regressed the IYCF indicators on HAZ and WHZ in separate models for each indicator. In models using stunting and wasting as the dependent variable, we present odds ratios, $95 \%$ confidence intervals and $P$-values from logistic regression models. In the Cambodia analysis, stunting was the only outcome variable assessed, and in the Ethiopia and Zambia analyses, only HAZ and WHZ were assessed. In all other countries, data were reported from analyses using all four outcome variables. Analyses from all countries used sample weights to adjust standard errors for the complex DHS survey design.

Data are presented only for models that adjusted for potentially confounding variables of the relation- 
ship between IYCF practices and child anthropometry. The included covariates differed across country data sets. All studies included child age, child sex and an asset-based wealth measure in models, and all studies, but Cambodia, included maternal education. The DHS does not measure household income or expenditures, but rather calculates an asset-based measure of wealth status using data on household assets, utility services, ownership of agricultural land and employment of domestic servants (Rutstein \& Johnson 2004). Household assets are assigned weights generated through principal component analysis, and the resulting asset scores are standardised and used to define population wealth quintiles.

All studies, but Cambodia and Uganda, also included maternal height, maternal body mass index, and maternal antenatal care attendance. Additional covariates used in models by country include the square of child age (Bangladesh, Kenya, Zimbabwe, India), current BF status (Bangladesh, Haiti, India, Kenya, Zimbabwe), urban vs. rural residence (Bangladesh, Haiti, Kenya, Zimbabwe), regional dummy variables (Bangladesh, Ethiopia, Haiti, India, Zambia), number of children (Bangladesh, India, Kenya, Zimbabwe), age of mother at first birth (Bangladesh, India, Kenya, Zimbabwe), current age of mother (Cambodia), birth type (Haiti) and child diarrhoea in the past 2 weeks (Kenya, Uganda, Zimbabwe). The India analysis also included the following covariates not included in other studies: size of child at birth, maternal employment status in the 12 months preceding the survey date, frequency of reading newspaper or magazine, frequency of listening to radio, frequency of watching television, household head's social identity (caste/tribe) and religion, and maternal diet diversity.

We did not conduct a meta-analysis of the results from the identified studies because of the small number of studies identified and their focus on different child anthropometric measures. Previous authors have examined pooled results of these relationships across countries (Marriott et al. 2012). This study aims to examine country-specific relationships between the IYCF indicators and child anthropometry and conduct cross-country comparisons. Therefore, we present results as summary tables and make qualita- tive comparisons across studies. $P$-values $<0.05$ were considered statistically significant for associations between the IYCF indicators and anthropometric outcomes within each country.

\section{Results}

\section{Characteristics of the samples}

We identified eight relevant studies that reported data from a total of nine countries (five from sub-Saharan Africa, three from Asia and one from the Caribbean region); one study examined data from both Ethiopia and Zambia (Ali et al. 2012) and another examined two consecutive DHS data sets from Cambodia (Marriott et al.2010), although only analyses from the more recent 2005 DHS data set examined in that study are presented. Table 2 reports select sample characteristics from the sample populations in each of the studies.

The sample populations in all countries were predominantly rural. The Ethiopian sample had the highest percentage of rural households (93\%) and the lowest percentage was in Haiti $(66 \%)$. More than three-quarters of the mothers in Ethiopia received no formal education, while in all other countries except India, fewer than one-third of sample mothers never attended school. The proportion of women attending at least some secondary schooling was extremely low in Kenya and Ethiopia at less than 5\%, while in Zimbabwe and Bangladesh, this percentage was much larger (64\% and 47\%, respectively).

In all countries, approximately one-quarter or more of children aged less than 24 months were stunted, with nearly half of all children stunted in Ethiopia and Zambia (47\% and $45 \%$, respectively) (Table 3 ). In all studies that disaggregated data by age grouping, the percentage of stunted children increased with increasing age group: the proportion of stunted children aged 18-23 months was two to three times greater than that of children aged 6-11 months. In Ethiopia and Haiti, $17 \%$ and $13 \%$ of children aged $0-23$ months, respectively, were wasted, as were $21 \%$ of children aged 6-23 months in Bangladesh. This percentage was lower among children 0-23 months in Kenya, Zambia and Zimbabwe at $8 \%, 7 \%$ and $5 \%$, respectively. The 


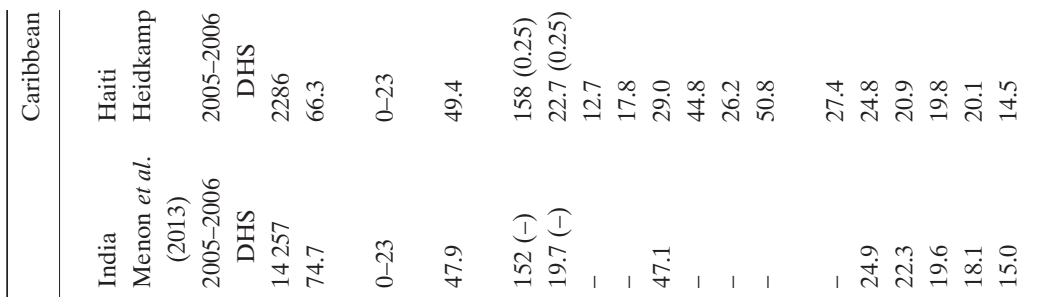

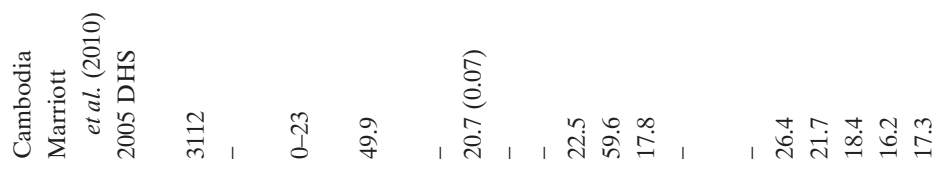

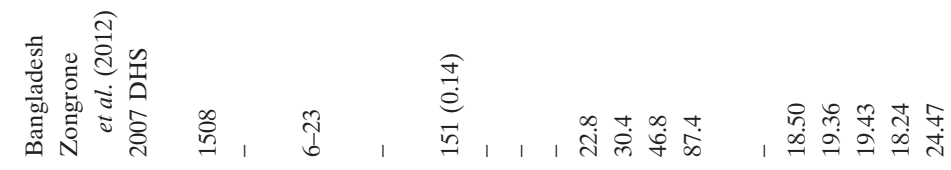

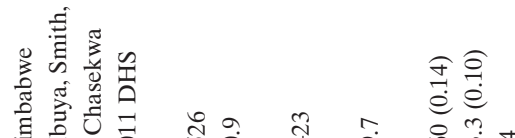

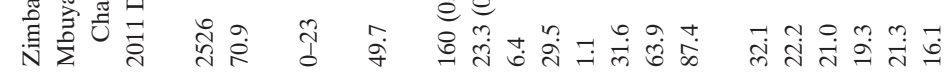

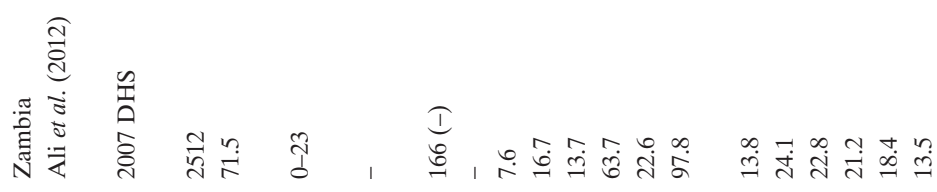

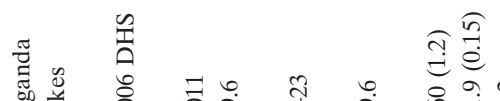

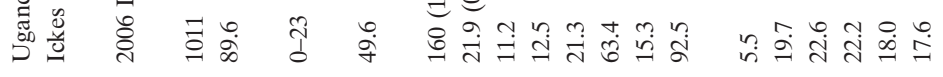

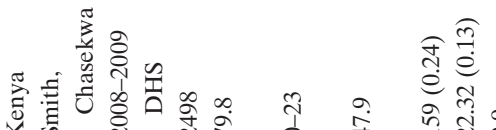

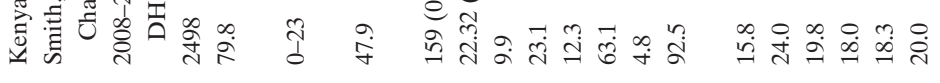

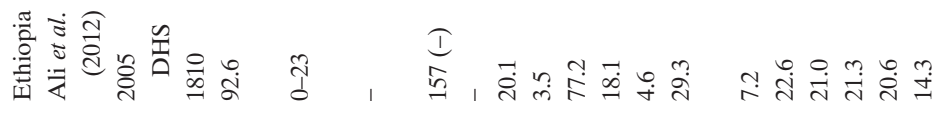
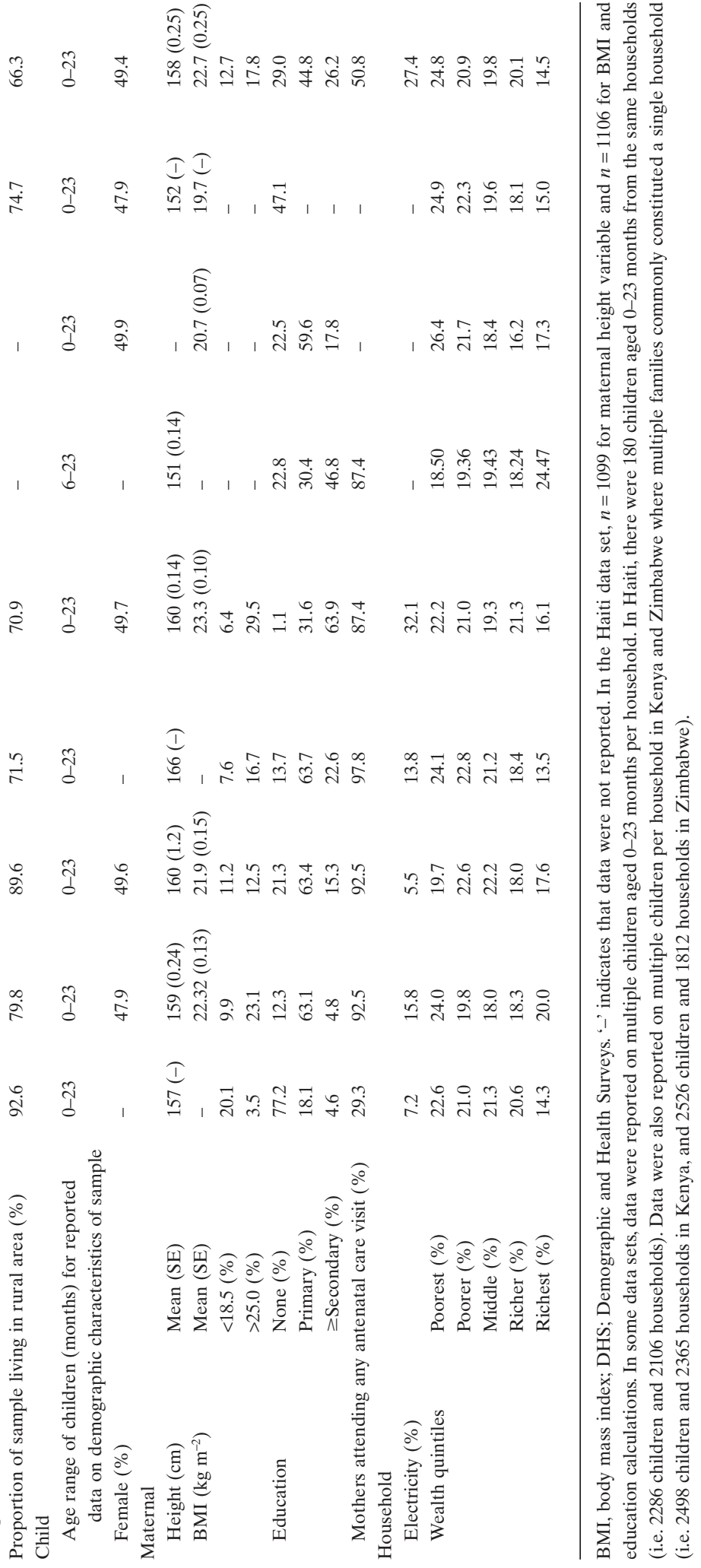


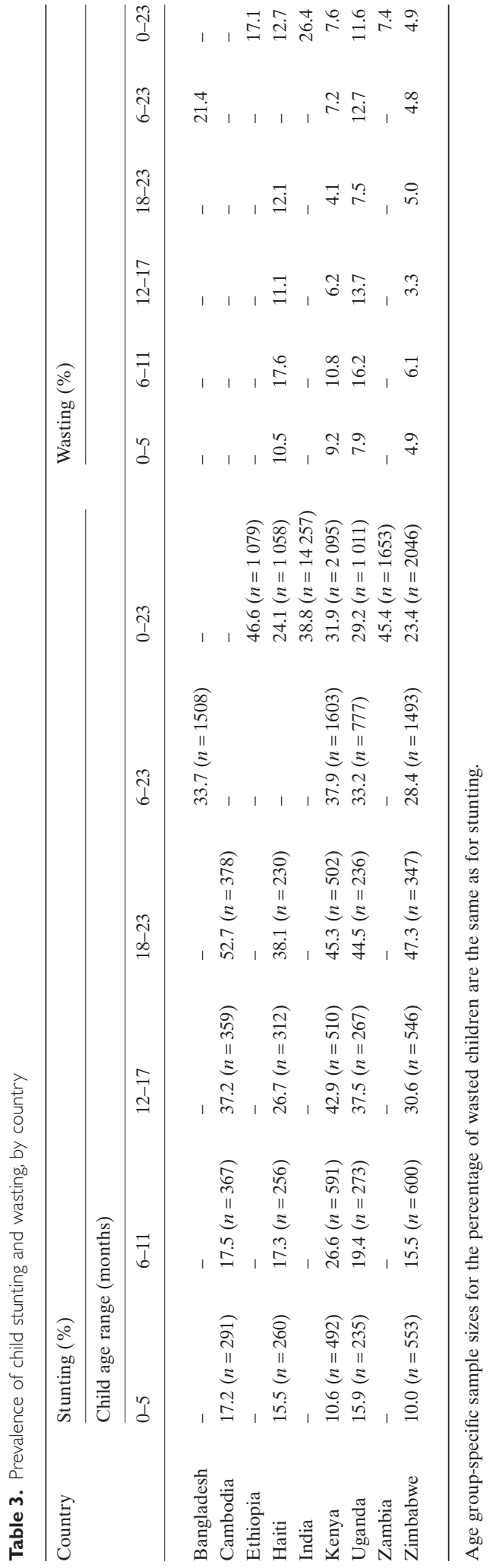

prevalence of wasting in children under 24 months was highest in India at $26 \%$.

\section{Breastfeeding and complementary feeding practices}

Approximately $40 \%$ of mothers in Bangladesh, Haiti and Uganda initiated BF of their youngest child within $1 \mathrm{~h}$ of birth (Table $4 \mathrm{a}$ ). More than half of mothers reported early BF initiation in Kenya and Zambia (58\% and 56\%, respectively), and this percentage reached $67 \%$ in Ethiopia and Zimbabwe. Only $23 \%$ of mothers reported initiating BF early in India. EBF under 6 months was lowest in Bangladesh at $36 \%$ and highest in Kenya at $78 \%$. At least four of five children aged 12-15 months in all countries received breast milk during the previous day, and approximately this same proportion of children aged 6-8 months received solid, semi-solid or soft foods during the previous day in all countries, except Ethiopia and India where fewer than two-thirds of children had received these foods (i.e. $61 \%$ and $64 \%$, respectively).

All studies, except for Cambodia, reported data on CF practices for children 6-23 months of age combined. Ethiopia demonstrated the poorest achievement of the WHO CF indicators, with only $7 \%$ of children receiving a minimally diverse diet during the previous day, $5 \%$ achieving the MAD indicator and $9 \%$ achieving the IRF indicator (Table $4 \mathrm{~b}$ ). Haiti, India, Uganda and Zimbabwe also showed a low proportion of children meeting these indicators (i.e. in India, Uganda and Zimbabwe, less than a quarter of children met the MDD indicator, and in all four countries, fewer than one in five children received a minimally acceptable diet) with somewhat higher percentages in Zambia, Bangladesh and Cambodia. Only in Bangladesh did the percentage of children aged 6-23 months that achieved the MAD indicator exceed $30 \%$.

The percent of children achieving the MMF indicator was considerably higher in all countries as compared with the MDD indicator, with more than half or nearly half of all children meeting this indicator in most countries. 


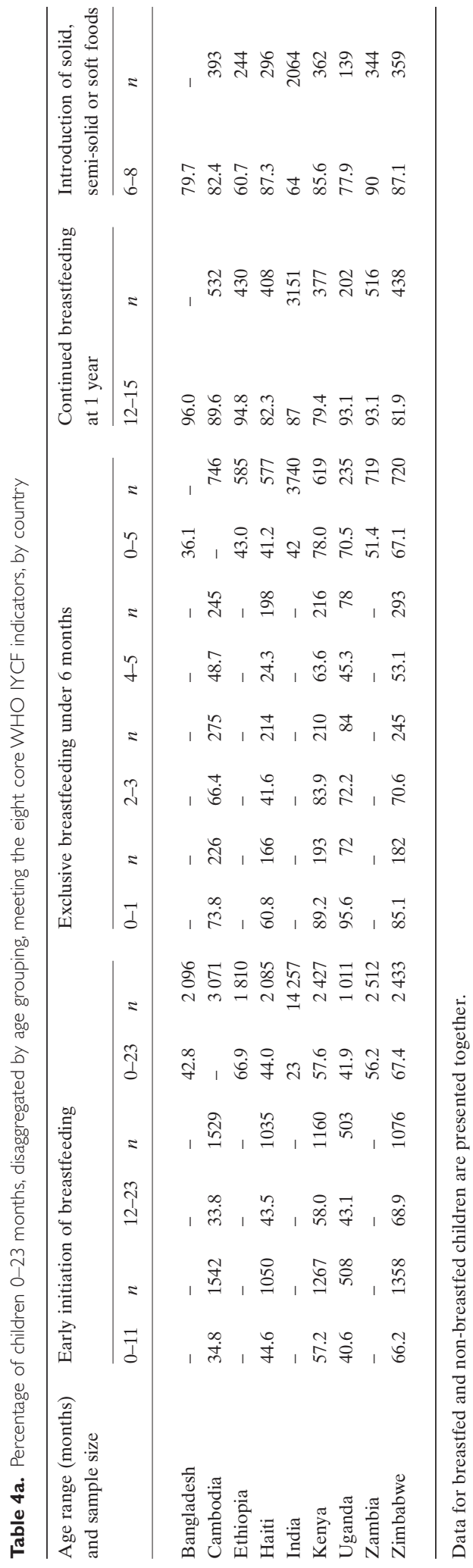

\section{Associations between WHO IYCF indicators and stunting/HAZ}

EIB was significantly positively associated with HAZ in Haiti $(P<0.05)$. EBF under 6 months was negatively associated with HAZ in seven of the data sets assessed; however, this association was significant only in Ethiopia and Kenya $(P<0.01$ and $P<0.05$, respectively) (Table 6). Continued $\mathrm{BF}$ at 1 year was also significantly negatively associated with $\mathrm{HAZ}$ in Ethiopia as well as Zimbabwe $(P<0.01$ and $P<0.05$, respectively), and demonstrated similar, although non-significant, trends in all other countries.

The ISF indicator was positively associated with HAZ in Bangladesh and Zambia $(P<0.01)$, and the odds of stunting were significantly lower for children 6-8 months of age in Bangladesh who achieved this indicator $(P<0.01)$ (Table 5). The MDD indicator was positively associated with HAZ in Bangladesh, India and Zambia $(P<0.05)$, and the odds of stunting were lower in children in India who achieved the MDD indicator $(P<0.001)$. Achieving the MAD indicator was similarly associated with a higher HAZ in these same three countries as well as in Ethiopia $(P<0.05)$. In Zimbabwe, the odds of stunting were significantly lower for children aged 6-23 months who met the MAD indicator $(P<0.05)$. The MMF and IRF indicators were not associated with HAZ or stunting in any of the data sets that assessed these relationships.

\section{Associations between WHO IYCF indicators and wasting/WHZ}

$\mathrm{EIB}, \mathrm{CBF}$ at 1 year, and the MDD and IRF indicators were not associated with wasting or WHZ in any of the data sets. Children in Bangladesh aged 0-5 months who received only breast milk the previous day were significantly less likely to be wasted $(P<0.05)$. The EBF indicator was also associated with higher WHZ in Bangladesh $(P<0.05)$ as well as in Zambia $(P<0.001)$ (Tables 5,6). Zambian and Zimbabwean children 6-8 months of age who were fed solid, semi-solid or soft foods the previous day showed significantly lower WHZ scores $(P<0.05)$. In Uganda, the odds of being wasted were significantly 


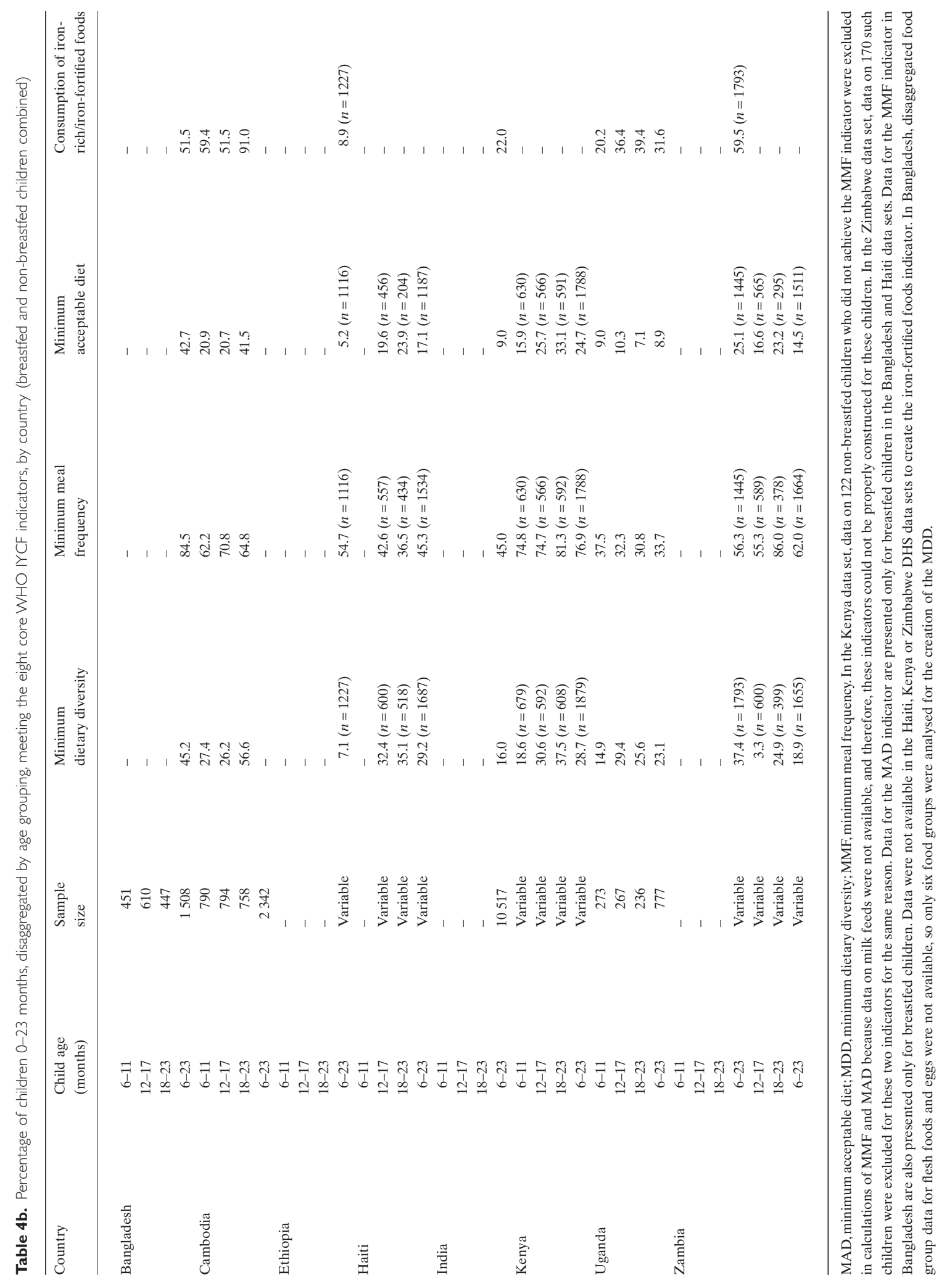




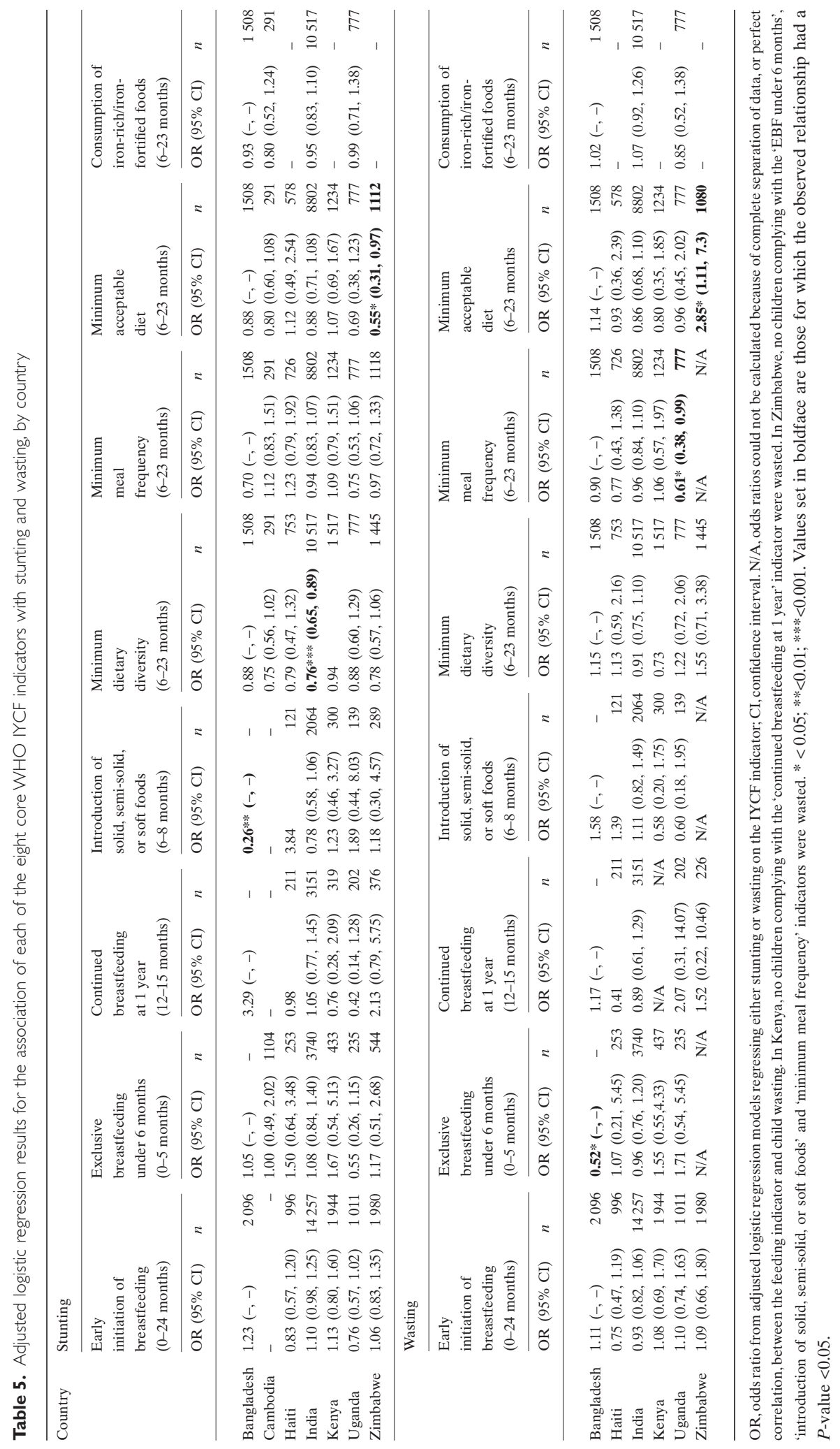




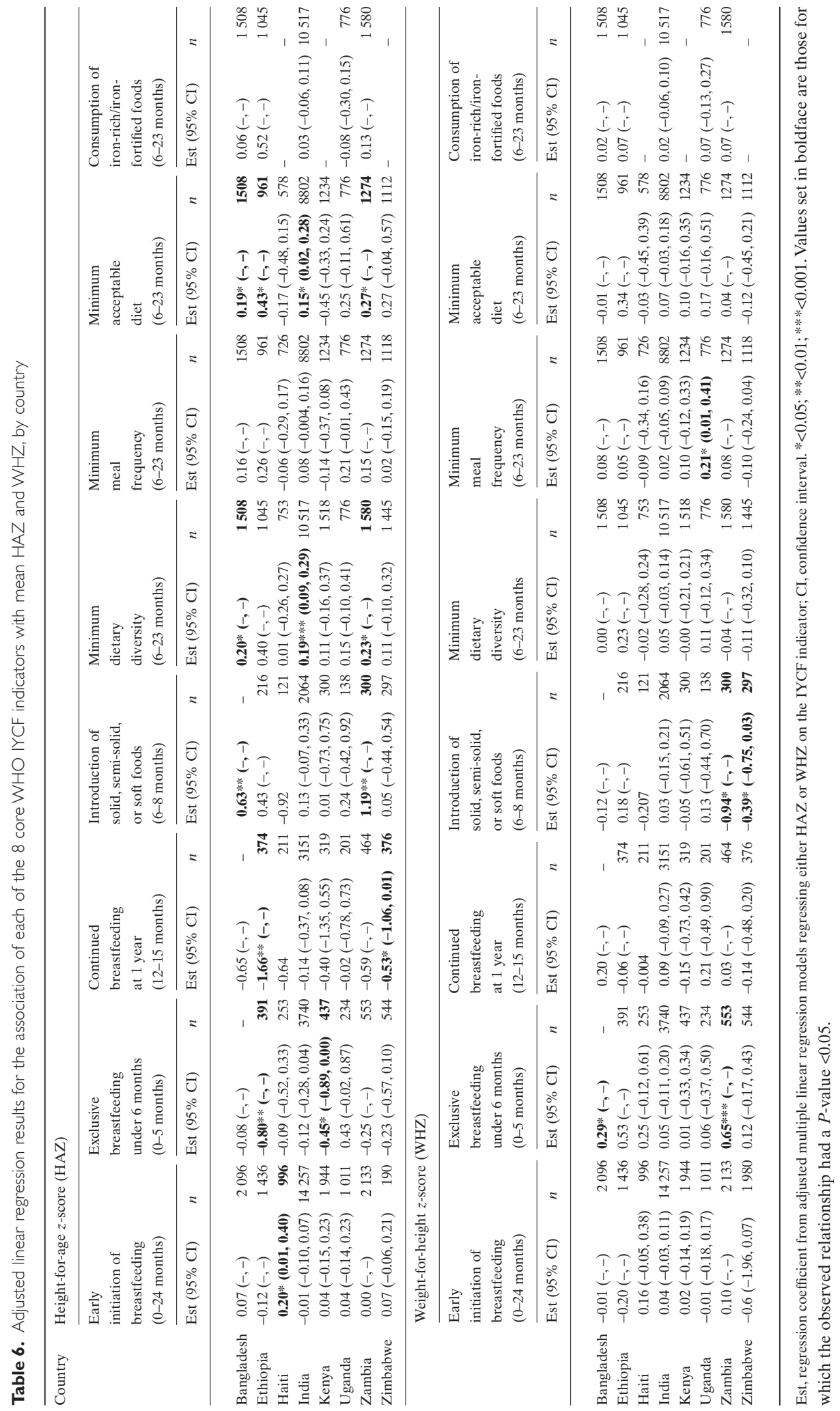


lower among children who met the MMF indicator, and there was a positive association observed between achievement of this indicator and WHZ $(P<0.05)$. In Zimbabwe, the odds of being wasted were significantly higher among children who met the MAD indicator $(P<0.05)$.

\section{Discussion}

The patterns of associations between the WHO IYCF indicators and child anthropometry varied widely across the country data sets examined. Among the BF indicators, EBF under 6 months was associated with lower odds of wasting in Bangladesh and higher WHZ in Bangladesh and Zambia. EBF under 6 months and $\mathrm{CBF}$ at 1 year were consistently negatively associated with HAZ. Of the CF indicators, the MAD indicator showed the strongest associations with HAZ (i.e. demonstrating significant positive relationships in four of the data sets) and the MDD indicator was positively associated with HAZ in Bangladesh, India and Zambia. The MMF and IRF indicators were not strongly associated with $\mathrm{HAZ}$ in any of the data sets. Child stunting was positively associated with the ISF indicator in Bangladesh, the MDD indicator in India and the MAD indicator in Zimbabwe, but was not associated with any other indicator in any country.

Child growth is determined by multiple factors, each is necessary but not sufficient. While the quality of child diets is certainly one of these determinants (Ruel \& Menon 2002), proper hygiene practices and access to adequate water, proper sanitation and reliable health services may be even more important determinants in some contexts. Yet, one might expect many of the IYCF practices that the WHO indicators aim to measure to be associated with child anthropometry. Considering first the $\mathrm{CF}$ indicators, dietary diversity is associated with child linear growth in many different contexts (Ruel \& Menon 2002; Arimond \& Ruel 2004; Rah et al. 2010). We would therefore expect the MDD and MAD indicators to show positive relationships with stunting and HAZ. To the extent that consumption of iron-rich foods is associated with consumption of animal-source foods or lipid-based nutrient supplements that are fortified with iron (e.g. Nutributter), we might also expect the IRF indicator to be associated with child linear growth (Rivera etal. 2003; Abu-Afarwuah et al. 2007). The MMF indicator is intended as a proxy for energy intake from non-breast-milk foods (World Health Organization 2008). If greater energy intakes result in more adequate nutrient intakes generally (Willett 2013), we might expect the MMF indicator to show positive associations with HAZ. However, adequate energy intakes alone are likely not sufficient to improve child linear growth (Allen 1994). The expected relationships between the BF indicators and child anthropometry are not as straightforward. Infants exclusively fed breast milk to 6 months do not show deficits in weight or length gain compared with infants exposed to mixed BF beginning at 3-4 months (Kramer \& Kakuma 2004). However, infants achieving the EBF indicator may not necessarily exhibit improved growth compared with non-EBF infants (Kramer et al. 2001). Longer duration of any BF has been shown to be associated with greater linear growth (Onyango et al. 1999; Simondon et al. 2001), although some studies have presented evidence to the contrary (Caulfield et al. 1996; Habicht 2000). Therefore, the relationship between the $\mathrm{CBF}$ indicator and child HAZ may depend, in part, on the quality of complementary foods fed at 1 year.

Therefore, these is evidence to suggest that the IYCF practices measured by the WHO indicators, especially the $\mathrm{CF}$ practices related to the overall quality of child diets, should demonstrate associations with the growth of young children. The WHO IYCF indicators though were designed as simple indicators that could be incorporated into large-scale surveys. These indicators are especially well suited for monitoring trends in diet quality in large-scale data sets wherein detailed dietary data cannot be collected; however, they may not be highly sensitive or specific measures of dietary quality in the analysis of the causal pathways to child growth. Although the relevance of these indicators does not depend in all cases on how well they are associated with child anthropometry at the individual level, understanding these associations is essential for selecting appropriate metrics for different purposes. We examine in more depth below the design of the individual 
indicators and their potential strengths and limitations in predicting anthropometric outcomes.

\section{Associations of WHO breastfeeding indicators with child anthropometry}

EBF under 6 months was negatively associated with HAZ in nearly all of the studies examined, and the association was statistically significant in Ethiopia and Kenya. The WHO EBF indicator, however, lacks sensitivity (i.e. it may commonly classify children as exclusively breastfed who may have received nonbreast-milk liquids or foods prior to the survey) and therefore overestimates the proportion of exclusively breastfed infants (World Health Organization 2008). This overestimation of EBF rates by 1-day recall measures has been observed previously (Zohoori et al. 1993; Piwoz et al. 1995; Arimond \& Ruel 2002). The lack of sensitivity of the EBF indicator could lead to the diminution of a potential positive association between EBF and HAZ. Alternatively, if the misclassification of infants aged $0-5$ months who are given non-breast-milk liquids and are growing more poorly is substantial, this misclassification could, in fact, lead to an observed negative association.

Socio-economic status (SES) is known to correlate with both child anthropometric outcomes (Adler \& Ostrove 1999; Ruel \& Menon 2002) and the practice of EBF (Lawoyin et al. 2001; Aidam et al. 2005). If not properly controlled for in analyses examining the association of EBF with child anthropometry, SES may confound the observed relationship. The DHS does not provide a stand-alone measure of SES, but rather an asset-based wealth measure. Aside from wealth, type of occupation and level of education are normally associated with SES. These two variables are not included in the DHS wealth measure so as to retain a pure measure of economic status and because these variables may independently influence health status (Rutstein \& Johnson 2004). All of the studies included in this synthesis included this same assetbased wealth measure as a covariate in regression analyses, and all but one study (i.e. Cambodia) included maternal education. Although it is possible that uncontrolled domains of SES (e.g. type of occupation) account for the negative associations observed between EBF and HAZ, this is unlikely given that a carefully developed measure of household wealth was included in models across all countries, maternal education was included as a covariate, and a consistent relationship was observed between the two variables across contexts. ${ }^{1}$

While EBF was negatively associated with HAZ, it was positively associated with WHZ in every country, with the relationship achieving statistical significance in Bangladesh and Zambia. This reverse trend has been observed previously (Trowbridge et al. 1987) and may be due, in part, to the statistical relationship between child height-for-age and child weight-forheight. As height is in the numerator and denominator of the two metrics, respectively, child linear growth spurts may lead to increases in height-for-age with simultaneous declines in weight-for-height (Golden 2009). Alternatively, WHZ may simply be a more responsive indicator of growth in this early infancy period as compared with HAZ - an indicator of chronic undernourishment for which cumulative growth deficits will be most pronounced later in infancy.

Consistent with previous studies (Caulfield et al. 1996; Marquis etal. 1997; Simondon et al. 2001; Sawadogo et al. 2006), findings from all eight data sets demonstrated a negative association between continued BF at 12-15 months and HAZ. This relationship reached statistical significance in Zimbabwe and Ethiopia where continued BF was associated with 0.53 and 1.66 SD lower HAZ, respectively. The authors have suggested that reverse causality underlies the negative associations observed between continued BF and HAZ, such that smaller, malnourished children, often seen as more vulnerable, are weaned later (Simondon et al. 2001; Habicht 2002). While the findings from the nine data sets reviewed here seem to support this reverse causality hypothesis, the crosssectional nature of these studies does not allow for causal inferences to be drawn from the observed relationships.

${ }^{1}$ Even in the India models where maternal employment was controlled for, EBF under 6 months showed a negative association with HAZ. 


\section{Association of WHO complementary feeding indicators with child anthropometry}

Introduction of solid, semi-solid or soft foods was positively associated with HAZ in Zambia and Bangladesh and reduced odds of stunting in Bangladesh. It was also associated, however, with lower child WHZ in Zambia and Zimbabwe. Because this indicator relies on a $24-\mathrm{h}$ recall period, similar to the $\mathrm{BF}$ indicators, it is plausible that children who received complementary foods prior to 6 months are, in fact, not excluded from the calculation of the indicator. On the other hand, children who are introduced to solid, semi-solid or soft foods in the 6-8 month window that the indicator examines may not have received those foods the day prior to the survey and therefore may also be misclassified. If so, the indicator will lack specificity and will not accurately reflect the timing of the initial introduction of solid, semi-solid or soft foods.

The MMF indicator, intended as a proxy for dietary energy intake, was positively associated with WHZ in Uganda, but was not strongly associated with any other anthropometric outcome. An appropriate number of daily feedings depends on the energy density of foods fed to children and the amount consumed at each feeding (PAHO/WHO 2003). The cutoffs as defined by the MMF indicator, therefore, would tend to underestimate the needs of children fed complementary foods of low energy density (i.e. approximately less than $0.8 \mathrm{kcal} \mathrm{g}^{-1}$; Dewey \& Brown 2003). On the other hand, as noted earlier, a high number of daily feedings of complementary foods may excessively displace breast milk and deleteriously affect child growth. Capturing these complex dynamics in a single indicator, amenable to largescale surveys may not be feasible and may contribute to the lack of association observed between the MMF indicator and child anthropometry.

In at least three of the countries where the MAD indicator was positively associated with HAZ, Bangladesh, India and Zambia, the relationship appears to be driven largely by the dietary diversity component of the MAD indicator. Previous studies have similarly observed the important role of dietary diversity in shaping relationships with child anthropometry when using summary child feeding measures (Arimond \& Ruel 2002; Sawadogo et al. 2006; Moursi et al. 2008).

The MDD indicator was significantly positively associated with HAZ in only three of the studies examined and was significantly negatively associated with stunting only in India. This finding was unexpected given the considerable evidence noted earlier, demonstrating the positive association between more diverse diets and better child growth. It is possible that the small percentage of children achieving the MDD indicator in some countries, Ethiopia in particular (Ali et al. 2012), did not allow for sufficient power to detect differences in child anthropometry. Alternatively, the consistent positive relationship observed between dietary diversity and household SES in different contexts (Ferguson et al. 1993; Hatløy et al. 2000; Hoddinott \& Yohannes 2002) may mean that controlling for SES in regression models that estimate the effects of dietary diversity on child anthropometry dampens the strength of the association observed between the two variables. Removing SES from models entirely, however, may lead to biased estimates if SES does not influence child anthropometry entirely through dietary diversity. Assessing multicollinearity and analysing regression models with and without controlling for household SES would help identify this as a potential complicating factor of such analyses.

An analysis of 10 data sets from Africa, Asia and Latin America that served as one input to the development of the WHO IYCF indicators found dietary diversity to be a useful indicator of dietary quality, with the mean micronutrient density adequacy $(\mathrm{MMDA})^{2}$ of diets increasing with increasing food group diversity (FANTA 2006). However, at a cut-off of four food groups, the dietary diversity indicator had a high sensitivity and low specificity for detecting a MMDA of less than $50 \%$ (i.e. the indicator would rarely misclassify an inadequate diet as adequate, but

${ }^{2}$ Mean micronutrient density adequacy is a measure of overall dietary quality, calculated as the mean of individual micronutrient density adequacies in the diet, each capped at $100 \%$. Individual micronutrient density adequacies are calculated as the percentage of the desired nutrient density for that age and breastfeeding status. 
would commonly misclassify adequate diets as inadequate). Such an indicator may be appropriate for identifying a population's potential to benefit from a dietary diversification intervention relative to other groups or relative to interventions emphasising other $\mathrm{CF}$ practices. The indicator may also be appropriate for assessing the adequacy of programs (Habicht et al. 1999) by providing a measuring stick to assess how well programme activities meet expected objectives. However, its frequent misclassification of adequate diets may make it less well suited in evaluation research settings where the impact of interventions on dietary quality or the causal pathway from child diet to growth must be rigorously assessed.

\section{Limitations}

This synthesis of findings has several limitations. Although the studies reviewed do not make causal claims, the data presented are cross sectional and the directions of the relationships observed are only hypothesised to be in the stated directions (i.e. feeding practices influence child anthropometry). The causal sequencing of these relationships cannot be determined, however, from the available data. In addition, assessing the associations between model covariates and child anthropometric outcomes across studies was beyond the scope of this synthesis. A fuller treatment of the associations of these potentially confounding variables with child anthropometric outcomes would be useful in future synthesis work. Finally, the studies reviewed were remarkably similar in both their standardised construction of the $\mathrm{WHO}$ indicators and their analysis approach. Yet, the different DHS questionnaires used in Kenya and Zimbabwe, differences in model covariates and the minor differences in construction of the CF indicators between countries (Table 4b) may limit, to some extent, the comparability of findings across countries.

\section{Conclusion}

The WHO IYCF indicators are clearly valuable tools in both programme and research settings for broadly assessing the quality of child diets and for monitoring population trends in IYCF practices over time. Their development has also served to remove a barrier to scaling up nutrition programming centred on child feeding by providing simple metrics for monitoring such programmes. This simplicity, however, means that the indicators necessarily simplify a great deal of the complexity inherent to child feeding, especially CF. For research and programmes that require analysis of how the quality of child diets is influenced by upstream determinants or how it affects downstream outcomes such as child growth, stronger proxies of dietary quality might be needed to discern these relationships (e.g. feeding scores or measures based on 7 -day food frequency recall data). Further research, in diverse contexts, examining the relationship of these indicators to other metrics of dietary quality and child nutritional status may be beneficial to programme planners, researchers and policy-makers in determining how best to apply them in specific contexts.

\section{Acknowledgements}

We would like to acknowledge Tawanda W. Ndapwadza for his contributions to the preparation and analysis of the Zimbabwe data set. We are also grateful to Jean-Pierre Habicht for his thoughtful comments on earlier drafts of this manuscript.

\section{Source of funding}

None.

\section{Conflicts of interest}

The authors declare that they have no conflicts of interest.

\section{Contributions}

SBI conducted the analysis of the Uganda data set. LES, MNNM and BC conducted the analysis of the Zimbabwe data set. LES also conducted the analysis of the Kenya data set. RAH conducted the analysis of the Haiti data set. PM conducted the analyses of the India, Zambia and Ethiopia data sets. AAZ conducted the analysis of the Bangladesh data set. ADJ synthesized the analyses. RJS and PM conceptualized the manuscript. The manuscript was written by ADJ 
and edited by SBI, LES, MNNM, RAH, PM, RJS, AAZ, and ADJ.

\section{References}

Abu-Afarwuah S., Lartey A., Brown K.H., Zlotkin S., Briend A. \& Dewey K.G. (2007) Randomized comparison of 3 types of micronutrient supplements for home fortification of complementary foods in Ghana: effects on growth and motor development. American Journal of Epidemiology 86, 412-420.

Adler N.E. \& Ostrove J.M. (1999) Socioeconomic status and health: what we know and what we don't. Annals of the New York Academy of Sciences 896, 3-15.

Aidam B.A., Pérez-Escamilla R., Lartey A. \& Aidam J. (2005) Factors associated with exclusive breastfeeding in Accra, Ghana. European Journal of Clinical Nutrition 59, 786-796.

Ali D., Rawat R., Subandoro A. \& Menon P. (2012) Infant and young child feeding (IYCF) practices in Ethiopia and Zambia and their association with child nutrition: analysis of demographic and health survey data. African Journal of Food, Agriculture, Nutrition and Development 12, 5895-5914.

Allen L.H. (1994) Nutritional influences on linear growth: a general review. European Journal of Clinical Nutrition 48 (Suppl. 1), S75-S89.

Arimond M. \& Ruel M.T. (2002) Progress in Developing an Infant and Child Feeding Index: An Example Using the Ethiopia Demographic and Health Survey 2000. International Food Policy Research Institute: Washington, DC.

Arimond M. \& Ruel M.T. (2004) Dietary diversity is associated with child nutritional status: evidence from 11 demographic and health surveys. The Journal of Nutrition 134, 2579-2585.

Arimond M., Daelmans B. \& Dewey K. (2008) Indicators for feeding practices in children. Lancet 371, 541-542.

Caulfield L.E., Bentley M.E. \& Ahmed S. (1996) Is prolonged breastfeeding associated with malnutrition? Evidence from nineteen demographic and health surveys. International Journal of Epidemiology 25, 693-703.

Dewey K.G. \& Begum K. (2011) Long-term consequences of stunting in early life. Maternal \& Child Nutrition $\mathbf{7}$, $5-18$.

Dewey K.G. \& Brown K.H. (2003) Update on technical issues concerning complementary feeding of young children in developing countries and implications for intervention programs. Food and Nutrition Bulletin 24, 5-28.

Engle P., Menon P. \& Haddad L. (1996) Care and Nutrition: Concepts and Measurement. International Food Policy Research Institute: Washington, DC.

Ferguson E.L., Gibson R.S., Opare Obisaw C., Osei Opare F., Lamba C. \& Ounpuu S. (1993) Seasonal food con- sumption patterns and dietary diversity of rural preschool Ghanaian and Malawian children. Ecology of Food and Nutrition 29, 219-234.

Food and Nutrition Technical Assistance Project (FANTA) (2006) Developing and Validating Simple Indicators of Dietary Quality and Energy Intake of Infants and Young Children in Developing Countries: Summary of Findings from Analysis of 10 Data Sets. Academy for Educational Development: Washington, DC.

Golden M.H. (2009) Proposed recommended nutrient densities for moderately malnourished children. Food and Nutrition Bulletin 30, S267-S342.

Habicht J.P. (2000) The association between prolonged breastfeeding and poor growth. In: Short and Long Term Effects of Breast Feeding on Child Health (eds B. Koletzko, K.F. Michaelsen \& O. Hernell), pp 193-200. Kluwer Academic/Plenum Publishers: New York.

Habicht J.P. (2002) The association between prolonged breastfeeding and poor growth - what are the implications? Advances in Experimental Medicine and Biology 478, 193-200.

Habicht J.P. \& Pelletier D.L. (1990) The importance of context in choosing nutritional indicators. The Journal of Nutrition 120 (Suppl. 11), 1519-1524.

Habicht J.P., Victora C.G. \& Vaughan J.P. (1999) Evaluation designs for adequacy, plausibility and probability of public health programme performance and impact. International Journal of Epidemiology 28, 10-18.

Hatløy A., Hallund J., Diarra M.M. \& Oshaug A. (2000) Food variety, socioeconomic status and nutritional status in urban and rural areas in Koutiala (Mali). Public Health Nutrition 3, 57-65.

Hoddinott J. \& Yohannes Y. (2002) Dietary Diversity as a Food Security Indicator. International Food Policy Research Institute: Washingon, DC.

ICF Macro (2011) Demographic and Health Surveys Methodology: Interviewer's Manual. ICF Macro: Calverton, MD.

Kramer M.S. \& Kakuma R. (2004) The optimal duration of exclusive breastfeeding: a systematic review. Advances in Experimental Medicine and Biology 554, 63-77.

Kramer M.S., Chalmers B., Hodnett E.D., Sevkovskaya Z., Dzikovich I., Shapiro S. et al. (2001) Promotion of breastfeeding intervention trial (PROBIT): a randomized trial in the Republic of Belarus. Journal of the American Medical Association 285, 413-420.

Lawoyin T.O., Olawuyi J.F. \& Onadeko M.O. (2001) Factors associated with exclusive breastfeeding in Ibadan, Nigeria. Journal of Human Lactation 17, 321325.

Marquis G.S., Habicht J.P., Lanata C.F., Black R.E. \& Rasmussen K.M. (1997) Association of breastfeeding and stunting in Peruvian toddlers: an example of 
reverse causality. International Journal of Epidemiology 26, 349-356.

Marriott B.P., White A.J., Hadden L., Davies J.C. \& Wallingford J.C. (2010) How well are infant and young child World Health Organization (WHO) feeding indicators associated with growth outcomes? An example from Cambodia. Maternal \& Child Nutrition 6, 358-373.

Marriott B.P., White A., Hadden L., Davies J.C. \& Wallingford J.C. (2012) World Health Organization (WHO) infant and young child feeding indicators: associations with growth measures in 14 low-income countries. Maternal \& Child Nutrition 8, 354-370.

Menon P., Bamezai A., Subandoro A., Ayoya M.A. \& Aguayo V. (2013) Age-appropriate infant and young child feeding practices are associated with child nutrition in India: insights from nationally-representative data. Maternal \& Child Nutrition doi: 10.111/mcn.12036.

Moursi M.M., Martin-Prevel Y., Eymard-Duvernay S., Capon G., Treche S., Maire B. \& Delpeuch F. (2008) Assessment of child feeding practices using a summary index: stability over time and association with child growth in urban Madagascar. The American Journal of Clinical Nutrition 87, 1472-1479.

Onyango A.W., Esrey S.A. \& Kramer M.S. (1999) Continued breastfeeding and child growth in the second year of life: a prospective cohort study in western Kenya. Lancet 354, 2041-2045.

PAHO/WHO (2003) Guiding Principles for Complementary Feeding of the Breastfed Child. Pan American Health Organization/World Health Organization: Washington, DC.

Piwoz E.G., Creed De Kanashiro H., Lopez De Romana G., Black R.E. \& Brown K.H. (1995) Potential for misclassification of infants' usual feeding practices using 24-hour dietary assessment methods. The Journal of Nutrition 125, 57-65.

Rah J.H., Akhter N., Semba R.D., de Pee S., Bloem M.W., Campbell A.A. et al. (2010) Low dietary diversity is a predictor of child stunting in rural Bangladesh. European Journal of Clinical Nutrition 64, 1393-1398.

Rivera J.A., Hotz C., González-Cossío T., Neufeld L. \& García-Guerra A. (2003) The effect of micronutrient deficiencies on child growth: a review of results from community-based supplementation trials. Journal of Nutrition 133, 4010S-4020S.

Ruel M.T. \& Menon P. (2002) Child feeding practices are associated with child nutritional status in Latin America: innovative uses of the demographic and health surveys. The Journal of Nutrition 132, 1180-1187.

Rutstein S.O. \& Johnson K. (2004) The DHS Wealth Index. DHS Comparative Reports No. 6. ORC Macro: Calverton, MD.

Savy M., Martin-Prevel Y., Sawadogo P., Kameli Y. \& Delpeuch F. (2005) Use of variety/diversity scores for diet quality measurement: relation with nutritional status of women in a rural area in Burkina Faso. European Journal of Clinical Nutrition 59, 703-716.

Sawadogo P.S., Martin-Prevel Y., Savy M., Kameli Y., Traissac P., Traore A.S. et al. (2006) An infant and child feeding index is associated with the nutritional status of 6- to 23-month-old children in rural Burkina Faso. The Journal of Nutrition 136, 656-663.

Simondon K.B., Costes R., Delaunay V., Diallo A. \& Simondon F. (2001) Children's height, health and appetite influence mothers' weaning decisions in rural Senegal. International Journal of Epidemiology 30, 476481.

Trowbridge F.L., Marks J.S., Lopez De Romana G., Madrid S., Boutton T.W. \& Klein P.D. (1987) Body composition of Peruvian children with short stature and high weight-for-height. II. Implications for the interpretation for weight-for-height as an indicator of nutritional status. The American Journal of Clinical Nutrition 46, 411-418.

UNICEF (2006) Nutrition Indicators: Exclusive Breastfeeding. A Report Card on Nutrition. http://www.unicef.org. (Number 4). [Online]. Available at: http://www.unicef. org/progressforchildren/2006n4/index_breastfeeding.html (Accessed 18 September 2012).

Willett W.C. (2013) Nutritional Epidemiology. Oxford University Press: New York, NY.

World Health Organization (1991) Indicators for Assessing Breastfeeding Practices. Division of Child Health and Development, World Health Organization: Geneva, Switzerland.

World Health Organization (2002) Report of Informal Meeting to Review and Develop Indicators for Complementary Feeding. 3-5 December. World Health Organization. Department of Child and Adolescent Health and Development and Department of Nutrition for Health and Development: Geneva, Switzerland.

World Health Organization (2008) Indicators for Assessing Infant and Young Child Feeding Practices: Part1 Definitions. World Health Organization: Geneva, Switzerland.

World Health Organization (2010a) Indicators for Assessing Infant and Young Child Feeding Practices: Part 3 Country Profiles. World Health Organization: Geneva, Switzerland.

World Health Organization (2010b) The WHO Child Growth Standards. World Health Organization: Geneva, Switzerland.

Zohoori N., Popkin B.M. \& Fernandez M.E. (1993) Breastfeeding patterns in the Philippines: a prospective analysis. Journal of Biosocial Science 25, 127-138.

Zongrone A., Winskell K. \& Menon P. (2012) Infant and young child feeding practices and child undernutrition in Bangladesh: insights from nationally representative data. Public Health Nutrition 15, 1697-1704. 